\title{
A liderança do enfermeiro no contexto dos serviços de urgência e emergência
}

\author{
The nurse's leadership within the context of emergency care services
}

Liderazgo del enfermero en el contexto de los servicios de urgencias y emergencias

Danielle Soares Silva ${ }^{1}$, Andrea Bernardes ${ }^{2}$, Carmen Silvia Gabriel ${ }^{3}$, Fernanda Ludmilla Rossi Rocha ${ }^{4}$, Graziela Caldana ${ }^{5}$

${ }^{1}$ Enfermeira. Ribeirão Preto, São Paulo, Brasil. E-mail: danielle.soares.silva@usp.br.

2 Enfermeira, Doutora em Enfermagem Fundamental. Professora Doutora da Escola de Enfermagem de Ribeirão Preto da Universidade de São Paulo

(EERP/USP). Ribeirão Preto, São Paulo, Brasil. E-mail: andreab@eerp.usp.br.

${ }^{3}$ Enfermeira, Doutora em Enfermagem Fundamental. Professora Doutora da EERP/USP. Ribeirão Preto, São Paulo, Brasil. E-mail: cgabriel@eerp.usp.br.

${ }^{4}$ Enfermeira, Doutora em Enfermagem. Professora Doutora da EERP/USP. Ribeirão Preto, São Paulo, Brasil. E-mail: ferocha@eerp.usp.br.

${ }_{5}^{5}$ Discente do curso de graduação em Enfermagem da EERP/USP. Ribeirão Preto, São Paulo, Brasil. E-mail: graziela.caldana@hotmail.com.

\section{RESUMO}

O estudo objetivou analisar as contribuições das pesquisas sobre liderança em enfermagem no contexto dos serviços de urgência/emergência no período de 2001 a 2012. Trata-se de Revisão Integrativa da Literatura, sendo incluídos estudos indexados no banco de dados da Literatura Latino-Americana e do Caribe em Ciências da Saúde (LILACS), Medical Literature Analysis and Retrieval Systems Online (MEDLINE) e SCOPUS. As publicações foram agrupadas em três categorias: "Estilos de liderança exercidos por enfermeiros de unidade de emergência"; "A liderança como estratégia para melhoria do gerenciamento da assistência de enfermagem"; "O desenvolvimento da liderança do enfermeiro no serviço de urgência e emergência". Grande parte das publicações tem baixo nível de evidência e está indexada em periódicos internacionais, revelando a necessidade de investimento pela comunidade científica nacional. Conclui-se que as teorias mais utilizadas pelos enfermeiros são: situacional e transformacional. É necessário investimento na comunicação e formação dos enfermeiros para o exercício da liderança.

Descritores: Liderança; Socorro de Urgência; Enfermagem em Emergência; Pesquisa em Administração de Enfermagem; Gerenciamento da Prática Profissional.

\section{ABSTRACT}

The objective of this study was to analyze the contributions that research has made to leadership in nursing within the context of emergency care services from 2001 to 2012. This Integrative Literature Review included studies indexed in the following databases: Latin American and Caribbean Health Sciences (LILACS), Medical Literature Analysis and Retrieval Systems Online (MEDLINE) and SCOPUS. Publications were grouped into three categories: "The styles of leadership adopted by the nurses of the emergency unit"; "Leadership as a strategy to improve nursing care management"; "The development of the nurses' leadership in emergency care services". A large part of the publications have a poor level of evidence and is indexed in international journals, showing that there is need for investments from both national and international scientific communities. In conclusion, the most commonly used theories among the nurses are: situational and transformational. Larger investments are necessary in communication and leadership training for nurses.

Descriptors: Leadership; Emergency Relief; Emergency Nursing; Nursing Administration Research; Practice Management.

\section{RESUMEN}

Se objetivó analizar contribuciones de investigaciones sobre liderazgo en enfermería en el contexto de los servicios de urgencias/emergencias, de 2001 a 2012. Revisión Integrativa de la Literatura, incluyéndose estudios indexados en los bancos de datos Literatura Latinoamericana y del Caribe en Ciencias de la Salud (LILACS), Medical Literature Analysis and Retrieval Systems Online (MEDLINE) y SCOPUS. Las publicaciones fueron agrupadas en tres categorías: "Estilos de liderazgo ejercidos por enfermeros de unidad de emergencias"; "Liderazgo como estrategia para mejora del gerenciamiento de la atención de enfermería"; "Desarrollo del liderazgo del enfermero en los servicios de urgencias y emergencias". Gran parte de las publicaciones posee bajo nivel de evidencia y está indexada en publicaciones internacionales, demostrando necesidad de inversiones de la comunidad científica nacional. Se concluye en que las teorías más utilizadas por los enfermeros son: situacional y transformacional. Es necesario invertir en comunicación y formación del enfermero para ejercer el liderazgo.

Descriptores: Liderazgo; Socorro de Urgencia; Enfermería de Urgencia; Investigación en Administración de Enfermería; Gestión de la Práctica Profesional. 


\section{INTRODUÇÃo}

A partir da instituição da Rede de Atenção às Urgências, amplia-se o conceito de saúde exigindo a participação multiprofissional no atendimento. A urgência é caracterizada por uma ocorrência imprevista de agravo à saúde com ou sem risco potencial de vida, cujo portador necessita de assistência imediata. Já a emergência é a constatação de risco iminente de vida ou sofrimento intenso, instituída por meio de práticas clínicas cuidadoras ${ }^{(1-}$ 2). Os departamentos de emergência são, portanto, locais que necessitam dar respostas rápidas, devendo ter uma equipe qualificada, que tenha facilidade de comunicação e capacidade de tomar decisões assertivas, uma vez que irá prestar cuidados de enfermagem de maior complexidade técnica a pacientes graves.

Esse conceito ampliado de saúde contribuiu para direcionar a intervenção e resposta às necessidades de saúde, atuando desde a promoção e prevenção, passando

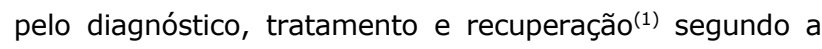
lógica do Sistema Único de Saúde (SUS), devendo contemplar todos os níveis de complexidade dessa Rede de Atenção.

A atenção primária à saúde, que constitui o componente pré-hospitalar fixo, tem um papel fundamental na estruturação e organização da rede de urgência e emergência do SUS, uma vez que funciona como a porta de entrada para os usuários. Já as redes de atenção pré-hospitalar móveis, constituem-se do Serviço de Atendimento Móvel de Urgências (SAMU-192) e os serviços associados de salvamento e resgate ${ }^{(3)}$, que juntamente com as Unidades de Pronto-Atendimento (UPAS), integram o nível intermediário de atenção às emergências. Com isso, o SAMU-192 e as UPAS estabelecem um importante elo entre os níveis de atenção à saúde, pois proporcionam uma adequada reorganização e reorientação dos usuários.

Os serviços de maior densidade tecnológica dizem respeito aos hospitais de atenção às urgências, bem como toda a gama de leitos de internação, passando pelos leitos gerais e especializados de retaguarda, de longa permanência e os de terapia semi-intensiva e intensiva. $\mathrm{E}$ por fim, o componente pós-hospitalar, que compõe as modalidades de atenção domiciliar, hospitais-dia e projetos de reabilitação integral com componente de reabilitação de base comunitária(3).

Em todos os níveis de atenção, a enfermagem desempenha um papel fundamental como integrante da equipe que presta atendimento de urgência, tanto no cuidado direto ao paciente, no gerenciamento do local e de toda sua equipe, como na educação permanente.
O trabalho em uma unidade de urgência é dinâmico, sendo que a equipe de saúde envolvida é que determina a competência funcional de resposta, de modo que os médicos e enfermeiros exercem papéis imperativos nesse atendimento $^{(4)}$. Nesse cenário, a liderança exerce um papel fundamental, pois é a partir dela que se obtém a sincronia do trabalho em equipe, um atendimento de qualidade, diminuição dos erros médicos e de enfermagem, gerando melhores resultados para o paciente $^{(5)}$.

A liderança é definida como a arte de influenciar pessoas a cumprir uma determinada tarefa e alcançar um objetivo em comum, utilizando-se das melhores estratégias, mantendo uma visão de futuro e sendo inovador. No contexto clínico, é caracterizada pela influência constante da equipe para a melhoria do cuidado prestado ao paciente(6). Assim, um bom líder é capaz de criar empatia, construir confiança e ambientes de trabalho mais saudáveis que promovam a segurança do paciente e excelência no atendimento(7).

Nesse sentido, a liderança se torna uma ferramenta essencial no trabalho do enfermeiro de urgência e emergência, seja no exercício da arte de cuidar, como também no gerenciamento da equipe e de suas condições de trabalho.

Esta pesquisa justifica-se dada a importância da liderança para o desempenho de um trabalho de qualidade nos serviços de urgência e emergência. Nesse contexto, adotam-se as diferentes teorias e estilos de liderança como referencial teórico, uma vez que explicam como esse processo ocorre ${ }^{(8-9)}$. Contudo, as teorias de liderança mais contemporâneas, como a Teoria Transformacional e Situacional, são as que mais se aplicam no contexto dos serviços de urgência e emergência, devido à motivação e estímulo, maiores níveis de compromisso e desempenho que esse tipo de trabalho requer ${ }^{(10-11)}$.

Ao líder cabe estimular a criatividade dos liderados, criar vínculos e promover discussões coletivas, permitir que participem dos processos decisórios e desenvolver o trabalho em equipe, de forma a alcançar o sucesso nos atendimentos prestados. Assim, as teorias de liderança situacional e transformacional são dois estilos essenciais para o enfermeiro no contexto da urgência e emergência. Porém, nenhum desses estilos é perfeito para todas as situações, cabendo aos líderes o desenvolvimento de conhecimentos e habilidades para aplicá-las conforme necessário( ${ }^{(6)}$.

Neste estudo, a compilação de dados a respeito do tema reuniu informações, buscando facilitar o entendimento do assunto e discussões sobre o papel do profissional de 
enfermagem na liderança de equipes de urgência e emergência. Assim, interroga-se: Quais as contribuições provenientes das pesquisas sobre liderança de enfermagem nos serviços de urgência e emergência? Qual é o tipo de líder que atua nesses serviços? Em busca de respostas a tais questionamentos, o presente trabalho objetivou analisar as contribuições das pesquisas produzidas sobre liderança em enfermagem, no contexto dos serviços de urgência e emergência no período de 2001 até 2012.

\section{PROCEDIMENTO METOdOLÓGICO}

Para atender aos objetivos deste estudo, utilizou-se a Revisão Integrativa da Literatura que é um método de pesquisa que permite a busca, a avaliação crítica e a síntese das evidências disponíveis sobre o tema investigado com a finalidade de reunir e sintetizar resultados de pesquisas sobre um delimitado tema, de maneira sistemática(12). Para a elaboração dessa revisão, seis etapas foram percorridas: Identificação do tema e estabelecimento do problema; Seleção da amostra; Categorização dos estudos; Análise dos resultados; Apresentação e discussão dos resultados; Apresentação da revisão.

Como critérios de inclusão foram considerados artigos primários que abordaram a temática "liderança em enfermagem" e "urgência/emergência", indexados nas bases de dados da Literatura Latino-Americana e do Caribe em Ciências da Saúde (LILACS), Medical Literature Analysis and Retrieval Systems Online (MEDLINE) e SciVerse SCOPUS, publicados no período de 2001 a 2012, na língua portuguesa, inglesa e/ou espanhola.

Os critérios de exclusão foram: publicações em forma de teses, dissertações, monografias, livros, revisões de qualquer estilo e relatos de experiência.

Foram utilizadas diferentes estratégias de busca com descritores controlados e não controlados. Os descritores controlados utilizados foram "liderança" e "enfermagem em emergência", pois são termos extraídos de um vocabulário estruturado e organizado, selecionado a partir do Decs/Mech (Descritores em Ciências da Saúde)(13). Já os descritores não controlados referem-se àqueles que são sinônimos identificados a partir da leitura dos textos primários. Nesse caso, foram utilizados os termos "liderança em enfermagem", "enfermagem em urgência" e "enfermagem em urgência e emergência".

Os títulos e resumos de 164 artigos foram lidos, resultando em uma pré-seleção de 88 . Após a leitura dos estudos na íntegra, realizadas aos pares, 76 foram excluídos por não estarem relacionados ao tema ou por não se constituírem em artigos primários. Assim, 12 estudos foram selecionados, sendo um na base de dados LILACS, um no LILACS e MEDLINE/PubMed, quatro na base de dados MEDLINE/PubMed, um na base de dados MEDLINE/PubMed e SCOPUS e cinco na base de dados Scopus.

Foi utilizado um formulário para coleta de dados bibliográficos, no qual foram extraídas informações acerca da identificação dos autores, base de dados onde o artigo foi encontrado, objetivo do estudo, metodologia, tipo de pesquisa, resultados e conclusões e nível de evidência. A classificação do nível de evidência foi realizada usando-se critérios de avaliação estabelecidos entre um e sete: nível 1evidências provenientes de revisão sistemática ou metanálise de ensaios clínicos randomizados controlados; nível 2 evidências derivadas de pelo menos um ensaio clínico randomizado controlado bem delineado; nível 3 - evidências obtidas de ensaios clínicos bem delineados sem randomização; nível 4 - evidências provenientes de estudos de coorte e de caso-controle bem delineados; nível 5 evidências originárias de revisão sistemática de estudos descritivos e qualitativos; nível 6 - evidências derivadas de um único estudo descritivo ou qualitativo; nível 7 - evidências oriundas de opinião de autoridades e/ou relatório de comitês de especialistas ${ }^{(14)}$.

Procedeu-se a leitura exaustiva dos artigos com posterior categorização por conteúdo temático. Os estudos foram agrupados em três categorias a partir das conexões e relações apresentadas pelos mesmos, de forma que possibilitassem explicações e interpretações do tema investigado: "Estilos de liderança exercidos por enfermeiros de unidade de emergência", "A liderança como estratégia para melhoria do gerenciamento do cuidado" e "O desenvolvimento da liderança do enfermeiro no serviço de urgência e emergência".

\section{RESULTADOS}

Nas bases de dados MEDLINE/PubMed e SCOPUS foi localizada a maior parte das publicações $(91,7 \%)$. Os artigos foram publicados em 11 periódicos diferentes, porém pouco foi produzido nesse período. Além disso, não há número significativo de publicações recentes que subsidie uma discussão atual sobre a temática. Metade das publicações utilizou a abordagem metodológica quantitativa e a outra metade qualitativa.

Com a finalidade de facilitar a interpretação dos dados, as publicações foram agrupadas em três categorias descritas no Quadro 1. 
Quadro 1: Sistematização dos artigos encontrados conforme categoria, descritos pelo sobrenome do autor, título e ano correspondente, tipo de estudo, nível de evidência e desfecho.

\begin{tabular}{|c|c|c|c|c|}
\hline Categoria & Publicação & $\begin{array}{l}\text { Tipo de } \\
\text { Estudo }\end{array}$ & $\begin{array}{c}\text { Nível de } \\
\text { Evidência }\end{array}$ & Desfechos \\
\hline \multirow{3}{*}{$\begin{array}{l}\text { Estilos de liderança } \\
\text { exercidos por } \\
\text { enfermeiros de } \\
\text { unidade de } \\
\text { emergência }\end{array}$} & $\begin{array}{l}\text { Wehbe, Galvão. Enfermeiro } \\
\text { de unidade de emergência: } \\
\text { sua liderança com o } \\
\text { pessoal auxiliar de } \\
\text { enfermagem, } 2001^{(15)} \text {. }\end{array}$ & $\begin{array}{c}\text { Estudo } \\
\text { quantitativo- } \\
\text { descritivo }\end{array}$ & 6 & $\begin{array}{c}\text { A liderança exercida pelo enfermeiro } \\
\text { de unidade de emergência é ideal ao } \\
\text { nível de maturidade do pessoal } \\
\text { auxiliar. Os estilos E3 (compartilhar) } \\
\text { e/ou E4 (delegar) são os mais } \\
\text { adotados. }\end{array}$ \\
\hline & $\begin{array}{c}\text { Wehbe, Galvão. Aplicação } \\
\text { da liderança situacional em } \\
\text { enfermagem de } \\
\text { emergência. } 2005^{(16)} \text {. }\end{array}$ & $\begin{array}{c}\text { Estudo } \\
\text { quantitativo- } \\
\text { descritivo }\end{array}$ & 6 & $\begin{array}{c}\text { O estudo mostra que o estilo de } \\
\text { liderança mais adotado pelo } \\
\text { enfermeiro foi o E3 e E4, sugerindo } \\
\text { que os liderados pesquisados } \\
\text { apresentam nível de maturidade alto. } \\
\text { A eficácia da prática da liderança vai } \\
\text { depender da habilidade do enfermeiro } \\
\text { em adaptar o estilo de liderança ao } \\
\text { nível de maturidade do membro da } \\
\text { equipe de enfermagem. }\end{array}$ \\
\hline & $\begin{array}{l}\text { Raup. The impact of ED } \\
\text { nurse manager leadership } \\
\text { style on staff nurse turnover } \\
\text { and patient satisfaction in } \\
\text { academic health center } \\
\text { hospitals. } 2008^{(17)} \text {. }\end{array}$ & $\begin{array}{c}\text { Estudo } \\
\text { quantitativo- } \\
\text { descritivo }\end{array}$ & 6 & $\begin{array}{l}\text { O estudo conclui que em hospitais } \\
\text { onde é utilizada a liderança } \\
\text { transformacional pelos enfermeiros, } \\
\text { há uma tendência na redução das } \\
\text { taxas de rotatividade quando } \\
\text { comparado aos hospitais onde outros } \\
\text { estilos são utilizados. }\end{array}$ \\
\hline \multirow{5}{*}{$\begin{array}{l}\text { A liderança como } \\
\text { estratégia para } \\
\text { melhoria do } \\
\text { gerenciamento do } \\
\text { cuidado }\end{array}$} & $\begin{array}{l}\text { Johnson. Leadership in a } \\
\text { Time of Disaster: Being } \\
\text { prepared for new age } \\
\text { threats. } 2002^{(18)} \text {. }\end{array}$ & $\begin{array}{l}\text { Estudo de } \\
\text { caso - estudo } \\
\text { descritivo }\end{array}$ & 6 & $\begin{array}{c}\text { Em tempos de desastre os líderes de } \\
\text { enfermagem têm a responsabilidade de } \\
\text { manter pacientes no centro de atenção. } \\
\text { Enfermeiros dever ser envolvidos no } \\
\text { planejamento das ações de atendimento } \\
\text { nesses casos. }\end{array}$ \\
\hline & $\begin{array}{l}\text { Walrath, Tomallo-Bowman, } \\
\text { Maguire. Emergency } \\
\text { Department: improving } \\
\text { patient satisfaction. } \\
2004^{(19)} \text {. }\end{array}$ & $\begin{array}{l}\text { Estudo de } \\
\text { caso - estudo } \\
\text { descritivo }\end{array}$ & 6 & $\begin{array}{c}\text { Uma equipe interdisciplinar, incluindo o } \\
\text { enfermeiro líder, foi formada para } \\
\text { reduzir o tempo de espera no } \\
\text { Departamento de Emergência. Essa } \\
\text { iniciativa leva tempo e requer foco e } \\
\text { envolvimento de todas as unidades do } \\
\text { hospital. }\end{array}$ \\
\hline & $\begin{array}{l}\text { Linton, Farrell. Nurses' } \\
\text { perceptions of leadership } \\
\text { in an adult intensive care } \\
\text { unit: A phenomenology } \\
\text { study. } 2009^{(20)} \text {. }\end{array}$ & $\begin{array}{c}\text { Estudo } \\
\text { qualitativo- } \\
\text { descritivo }\end{array}$ & 6 & $\begin{array}{c}\text { Este estudo explorou a percepção de } \\
\text { liderança de enfermeiros da UTI adulto. } \\
\text { A análise dos dados revelou cinco } \\
\text { temas: liderar pelo exemplo; } \\
\text { comunicação; capacidade de pensar } \\
\text { diferentemente do gerente; } \\
\text { conhecendo o pessoal; e intensificação } \\
\text { na crise. }\end{array}$ \\
\hline & $\begin{array}{l}\text { Ritchie, Laschinger, Wong. } \\
\text { The effects of emotionally } \\
\text { intelligent leadership } \\
\text { behaviour on emergency } \\
\text { staff nurses' workplace } \\
\text { empowerment and } \\
\text { organizational commitment. } \\
2009^{(21)} \text {. }\end{array}$ & $\begin{array}{c}\text { Estudo } \\
\text { quantitativo }\end{array}$ & 4 & $\begin{array}{l}\text { Na visão dos enfermeiros, líderes } \\
\text { emocionalmente inteligentes têm forte } \\
\text { relação com o empoderamento e } \\
\text { tomada de decisões, influenciando no } \\
\text { comprometimento com a instituição e } \\
\text { na qualidade da assistência prestada. }\end{array}$ \\
\hline & $\begin{array}{l}\text { Wolf. Acuity Assignation: } \\
\text { An Ethnographic } \\
\text { Exploration of Clinical } \\
\text { Decision Making by } \\
\text { Emergency Nurses at Initial } \\
\text { Patient Presentation. } \\
2010^{(22)} \text {. }\end{array}$ & $\begin{array}{c}\text { Estudo } \\
\text { qualitativo- } \\
\text { descritivo }\end{array}$ & 6 & $\begin{array}{l}\text { Determinações da acuidade dos } \\
\text { pacientes foram influenciadas pela } \\
\text { liderança, comunicação, volume de } \\
\text { pacientes e tempo na triagem. Decisões } \\
\text { sobre a gravidade dependiam do } \\
\text { conhecimento do enfermeiro e do } \\
\text { contexto social. }\end{array}$ \\
\hline
\end{tabular}




\begin{tabular}{|c|c|c|c|c|}
\hline Categoria & Publicação & $\begin{array}{l}\text { Tipo de } \\
\text { Estudo }\end{array}$ & $\begin{array}{c}\text { Nível de } \\
\text { Evidência }\end{array}$ & Desfechos \\
\hline & $\begin{array}{l}\text { Adriaenssens, Gucht, Maes. } \\
\text { The impact of traumatic } \\
\text { events on emergency room } \\
\text { nurses: findings from a } \\
\text { questionnaire survey. } \\
2012^{(23)}\end{array}$ & $\begin{array}{c}\text { Estudo } \\
\text { transversal }\end{array}$ & 4 & $\begin{array}{l}\text { O oferecimento de suporte social pelo } \\
\text { líder e o estabelecimento de uma } \\
\text { cultura de apoio ao enfermeiro vítima } \\
\text { de eventos traumáticos afeta } \\
\text { positivamente esse enfermeiro, bem } \\
\text { como contribui com a qualidade da } \\
\text { assistência prestada. }\end{array}$ \\
\hline & $\begin{array}{c}\text { Johansen, Neweark. } \\
\text { Conflicting priorities: } \\
\text { emergency nurses' } \\
\text { perceived disconnect } \\
\text { between patient satisfaction } \\
\text { and the delivery of quality } \\
\text { patient care. } 2012(24)\end{array}$ & $\begin{array}{c}\text { Estudo } \\
\text { qualitativo }\end{array}$ & 6 & $\begin{array}{l}\text { Enfermeiros do Departamento de } \\
\text { Emergência sentem-se frustrados } \\
\text { quando líderes não ouvem suas } \\
\text { necessidades. Relatam que a } \\
\text { satisfação do paciente não é, } \\
\text { necessariamente, um indicador de } \\
\text { qualidade do atendimento. }\end{array}$ \\
\hline \multirow{2}{*}{$\begin{array}{l}\text { O desenvolvimento } \\
\text { da liderança do } \\
\text { enfermeiro no } \\
\text { serviço de urgência e } \\
\text { emergência }\end{array}$} & $\begin{array}{l}\text { Gilligan, Bhatarcharjee, } \\
\text { Knight, Smith, Hegarty, } \\
\text { Shenton, et al. To lead or } \\
\text { not to lead? Prospective } \\
\text { controlled study of } \\
\text { emergency nurses' } \\
\text { provision of advanced life } \\
\text { support team. } 2005^{(25)} \text {. }\end{array}$ & $\begin{array}{c}\text { Estudo } \\
\text { prospectivo } \\
\text { quantitativo }\end{array}$ & 4 & $\begin{array}{c}\text { Enfermeiros capacitados em suporte } \\
\text { avançado de vida conhecem as causas } \\
\text { potencialmente irreversíveis da parada } \\
\text { cardíaca, caso não sejam tomadas as } \\
\text { devidas providências em tempo hábil. } \\
\text { Assim, se um médico não está } \\
\text { disponível para liderar a equipe de } \\
\text { reanimação, é apropriado que } \\
\text { enfermeiro assuma o papel de líder da } \\
\text { equipe. }\end{array}$ \\
\hline & $\begin{array}{l}\text { Spivak, Smith, Logsdon. } \\
\text { Developing expert clinical } \\
\text { nurses: Grow them, hold } \\
\text { them and let them walk } \\
\text { away. } 2011^{(26)} \text {. }\end{array}$ & $\begin{array}{l}\text { Estudo de } \\
\text { caso - estudo } \\
\text { descritivo }\end{array}$ & 6 & $\begin{array}{c}\text { Enfermeiros clínicos do Departamento } \\
\text { de Emergência precisam de orientação } \\
\text { dos líderes acerca de seus } \\
\text { comportamentos no trabalho e se } \\
\text { sentem motivados quando esses } \\
\text { líderes estão familiarizados com suas } \\
\text { habilidades. }\end{array}$ \\
\hline
\end{tabular}

\section{DISCUSSÃO}

A existência de periódicos internacionais especializados na temática liderança na área da enfermagem pode justificar a localização do maior número de artigos indexados nas bases de dados MEDLINE/PubMed e SCOPUS. O pequeno número de publicações sobre a temática pesquisada em periódicos nacionais sugere investimento incipiente dos pesquisadores brasileiros acerca do assunto, ainda que se reconheça a importância da liderança para o trabalho do enfermeiro no contexto da urgência e emergência.

A partir da leitura dos artigos selecionados, foram criadas três categorias de análise. Na categoria "Estilos de liderança exercidos por enfermeiros de unidade de emergência" foram reunidos três artigos que visam identificar, além dos estilos de liderança exercidos por enfermeiros, os estilos de liderança que estes devem adotar frente ao perfil dos seus liderados. Além disso, visam investigar a influência das teorias e estilos de liderança adotados pelos enfermeiros da unidade de urgência/emergência na rotatividade do pessoal de enfermagem e satisfação do paciente no seu atendimento.

A Teoria Contingencial ou Situacional baseia-se na premissa de que não existe um único estilo de liderança apropriado para toda e qualquer situação. Essa Teoria foi descrita como 0 produto da relação entre 0 comportamento diretivo do líder e o nível de maturidade do liderado, sendo que esse comportamento representa o grau de direção dada ao grupo em termos de definições de papéis. Assim, os quatro estilos de liderança propostos por Hersey e Blanchard, idealizadores dessa teoria, são: determinar (E1), persuadir (E2), compartilhar (E3) e delegar $(E 4)^{(5)}$.

Estudos trazem como resultado que os estilos de liderança E3 e E4 apareceram como os mais adotados pelos enfermeiros da unidade de emergência, sendo estes também os estilos que eles deveriam adotar frente ao perfil dos liderados. Esses estilos dão autonomia ao liderado quanto às decisões a serem tomadas e o líder, neste caso, colabora somente quando ocorre alguma complicação(15-16).

Embora tais pesquisas tenham baixo nível de evidência (6), por se tratarem de estudos descritivos, elas trazem dados importantes para a prática do enfermeiro. É desejável que o profissional que trabalha com urgência e emergência encoraje os integrantes da equipe de enfermagem a tomarem decisões e que a comunicação seja bilateral, uma vez que o estímulo e a motivação permanecem elevados nesse caso $^{(15,27)}$. 
$O$ estilo E4 é o mais indicado para o enfermeiro que trabalha em sala de urgência, porém é essencial que a maturidade dos liderados também seja considerada: maturidade para o trabalho, relacionada com o conhecimento e capacidade técnica; e maturidade psicológica, que se refere à disposição ou motivação para realizar alguma coisa(28).

Os estudos destacados nesta categoria usaram como referencial teórico a Liderança Situacional, de modo que os estilos de liderança que mais se destacaram, têm como perspectiva a autonomia cada vez maior do liderado frente às atividades que desenvolvem no cenário dos serviços de urgência/emergência. Tendo em vista este panorama, torna-se fundamental que os profissionais da equipe de enfermagem estejam cada vez mais qualificados para o trabalho, objetivando maior autonomia e uma liderança eficaz por parte do enfermeiro. Para tanto, esse profissional deve desenvolver ao longo de sua carreira a competência de liderar, exercitando o autoconhecimento quanto aos estilos utilizados e os adequando às diferentes situações e nível de maturidade dos seus liderados.

Como estratégia para o desenvolvimento da liderança do enfermeiro, o ensino sobre liderança através da educação permanente/continuada hospitalar poderia ser uma proposta bastante interessante para a melhoria da qualidade na assistência de enfermagem nos serviços de urgência/emergência. A educação permanente em urgência é fundamental neste processo, pois ajusta a equipe de saúde às necessidades vigentes na prática, levando em conta as particularidades de cada região e de cada individuo, atendendo-o na sua especificidade. Um dos enfoques da Política Nacional de Educação Permanente em Saúde ${ }^{(29)}$ é incorporar o ensino e o aprendizado à vida cotidiana das organizações e às práticas sociais e laborais, no contexto real em que ocorrem.

Além disso, a inserção de conteúdos na grade curricular dos cursos de graduação em enfermagem que viabilizem o aprendizado sobre liderança, possibilitaria o melhor preparo dos futuros profissionais de enfermagem para atuarem no contexto da urgência/emergência.

A liderança transformacional e sua influência sobre a rotatividade do pessoal de enfermagem e satisfação do paciente no atendimento caracteriza-se como outra importante teoria abordada(17). Trata-se de uma teoria contemporânea que conduz os liderados a um alto nível de motivação, através de uma relação baseada na confiança, valorizando a criatividade e a inovação, de modo que o líder deve estar atento às preocupações e necessidades dos seus liderados(30-31). Estudos demonstram que os pacientes associam um aumento da satisfação no trabalho e redução de eventos adversos quando utilizada a liderança transformacional ${ }^{(32-33)}$.

Um líder transformacional é aquele que demonstra inspiração, motivação, influência da equipe, implementando abordagens criativas para a resolução dos problemas, criando oportunidades de aprendizagem e crescimento de cada trabalhador com base em suas habilidades e desejos. Além disso, esse líder influencia positivamente a sua equipe, demonstrando ações corretas diante de condições adversas, promovendo comportamentos éticos e morais de sua equipe, mantendo assim, a confiança do pessoal ${ }^{(17,34)}$.

Nesse sentido, o desenvolvimento dessa liderança em gestores permite que as organizações inspirem o trabalhador de forma positiva, resultando em um atendimento de qualidade ao paciente ${ }^{(32)}$, além da tendência na redução das taxas de rotatividade do pessoal de enfermagem ${ }^{(17)}$.

Tendo em vista que na área de urgência e emergência a equipe deve trabalhar de forma sincronizada, rápida e com segurança em prol de uma melhor qualidade da assistência de enfermagem, é essencial que haja a capacitação dos trabalhadores ${ }^{(35)}$. Dessa forma, a rotatividade do pessoal de enfermagem não é desejada, uma vez que os vínculos teriam que ser restabelecidos a cada troca de funcionário e a capacitação em suporte básico e avançado de vida ficaria comprometida.

\section{A categoria "A liderança como estratégia para a} melhoria do gerenciamento do cuidado" reuniu sete artigos sendo dois com nível de evidência intermediário (4) e os demais com baixo nível de evidência (6).

O enfermeiro necessita compreender o processo de liderar e desenvolver as competências necessárias, dentre elas, a comunicação, o relacionamento interpessoal, tomada de decisões, além da habilidade clínica para alcançar sucesso na assistência prestada. Alguns estudos mostram que os profissionais de serviços de urgência e emergência reconhecem a comunicação e a tomada de decisão como ferramentas de extrema importância para a garantia do sucesso na prestação dessa assistência aos pacientes que se encontram em situações de risco, porém destacam que há muitas dificuldades no estabelecimento desses processos(23-24,27-36). Importante ressaltar que a comunicação deve ser intensa e dialógica e que a tomada de decisão deve ser coletiva, de modo a manter a equipe motivada, assumindo a corresponsabilidade pelo alcance dos resultados desejados.

Além da comunicação, outros aspectos são importantes para se alcançar qualidade na prestação do cuidado em saúde, como por exemplo, a importância de se 
ter líderes de sucesso, de dar exemplo aos liderados, a habilidade de pensar fora do âmbito da gestão e de lidar com conflitos, além de ter uma visão ampliada do trabalho ${ }^{(19-20,24,37)}$. Conflitos são inevitáveis quando se atua em ambientes com grande demanda de trabalho. Saber lidar com eles é necessário para que se evitem consequências negativas, especialmente quando se vivencia situações estressantes e traumáticas ${ }^{(23-24)}$.

Especialmente em situações de catástrofes, como a ocorrida em setembro de 2001, a resolução de conflitos, a comunicação, a liderança, a coordenação, o trabalho em equipe e o envolvimento de todos no planejamento das ações são essenciais ${ }^{(18)}$. A classificação de risco dos pacientes em situação de urgência e emergência deve ser feita não só na ocorrência de catástrofes ou desastres, mas em qualquer situação em que o paciente necessite de atendimento rápido. Ratifica-se que a perspicácia e habilidade nesta avaliação são influenciadas pela comunicação adequada, tempo de espera reduzido no departamento de emergência e pela liderança exercida nessa unidade. $\mathrm{O}$ líder percebido como ineficiente reduz a credibilidade das diretivas para a mudança da prática(22).

Nesse contexto, não há dúvidas que a liderança eficiente deve ser usada como estratégia para a melhoria do gerenciamento do cuidado prestado ao paciente. Um dos atributos importantes dos líderes eficientes refere-se à inteligência emocional que se trata de uma habilidade dos líderes em lidar com suas próprias emoções e com as de outras pessoas. Além disso, destacam a importância de garantir aos enfermeiros o desenvolvimento de competências de liderança em sua formação, que incluam habilidades de relação interpessoal, aprimoramento da inteligência emocional e o conhecimento de estratégias para a criação de empoderamento do ambiente de trabalho ${ }^{(21,38)}$.

Em suma, é imprescindível que haja compromisso das instituições de ensino superior em enfermagem, de inserirem nos seus currículos conteúdos que viabilizem o aprendizado sobre liderança, formando assim enfermeiros mais bem preparados que possam oferecer uma assistência de melhor qualidade. As próprias Diretrizes Curriculares Nacionais para os Cursos de Graduação em Enfermagem definem a liderança como uma das competências do profissional enfermeiro, sendo que essa é uma das principais competências a serem adquiridas por todos os profissionais da saúde ${ }^{(39-40)}$.

O processo educativo voltado à liderança, bem como para lidar com o inesperado é essencial, uma vez que os profissionais atribuem um sentido que traduzem uma progressão em termos de qualificação dos processos de atendimento da demanda dos usuários nas urgências e emergências(18-19,41).

Na categoria "O desenvolvimento da liderança do enfermeiro no serviço de urgência e emergência" enquadraram-se dois artigos, sendo um com nível de evidência intermediário (4) e outro baixo (6).

Os estudos destacam a importância de se desenvolver nos profissionais que atuam em urgência e emergência a competência de liderar. Esse desenvolvimento é possível, visto que a liderança envolve habilidades pessoais e técnicas com componentes inatos e aprendidos ${ }^{(42-43)}$.

Como o enfermeiro, na maioria das vezes, é o membro da equipe que primeiro se depara com a situação de emergência, especialmente a parada cardiorrespiratória, este deve estar preparado para atuar com competência, iniciando as manobras básicas de reanimação, o que demanda tomada de decisão rápida e liderança dentro da equipe ${ }^{(44)}$.

Porém, um paradoxo parece existir no ato da ressuscitação cardíaca, pois muitos enfermeiros são instrutores em cursos de suporte avançado de vida, mas na realidade não atuam como líderes de equipe de reanimação. Isso parece sugerir que os enfermeiros têm competência suficiente para ensinar técnicas de suporte avançado de vida, mas não para liderar a equipe envolvida(25). Contudo, a liderança é um dos principais instrumentos do enfermeiro para a gerência dos processos de trabalho, coordenação e articulação das atividades em serviços de emergência que envolve a produção do cuidado e, conforme destacado, é uma atividade essencial a ser aprendida(45). $^{(45}$.

Algumas medidas podem ser adotadas por enfermeiros de emergência para desenvolver seu potencial de liderança, com a finalidade de melhorar o bem-estar de si mesmos e da sua equipe no ambiente estressante das emergências, bem como a qualidade no atendimento aos pacientes ${ }^{(46)}$.

Ao exercer uma liderança eficaz, o enfermeiro adquire respeito e confiança de sua equipe à medida que os resultados do seu trabalho são satisfatórios. Atrelado a este contexto, quando o enfermeiro exerce uma boa liderança, a comunicação entre os membros da equipe torna-se mais adequada e clara, reduzindo assim, os erros. Nesse sentido, esse profissional deve se tornar observador objetivo, levando em consideração as situações tanto de suas próprias perspectivas quanto de outras pessoas, pois este comportamento permitiria avaliar suas ações de forma crítica e refletir sobre a adequação dos diferentes estilos de liderança a cada situação, conferindo-lhe melhores resultados.

O conhecimento também deve ser colocado como importante para o desenvolvimento da liderança. Ao 
identificar lacunas no conhecimento, o enfermeiro deve buscar preenchê-las por meio de capacitação para o desenvolvimento da habilidade de liderar. O conhecimento teórico-prático e os processos e rotinas são essenciais para o exercício da liderança ${ }^{(30,46)}$.

Nessa perspectiva, são necessários investimentos nas diferentes competências, seja de comunicação ou de relacionamento interpessoal, além de tempo e esforços dedicados ao desenvolvimento de lideranças de enfermagem para atuarem nos serviços de urgência e emergência. Os enfermeiros especialistas precisam de orientação de líderes de enfermagem e estes últimos, quando estão bem familiarizados com as habilidades e motivações de seus empregados, são capazes de promover a saúde e melhorar a qualidade da assistência nas unidades.

Destaca-se que vários estudos que compõem as diferentes categorias têm baixo nível de evidência(14), trazendo à tona a necessidade de que sejam realizadas pesquisas que melhorem a objetividade e validade dos resultados. Somado a isso, há que se ressaltar a questão da lacuna de conhecimento do enfermeiro no que tange à liderança e a necessidade de intensificação da comunicação, fato que ratifica a importância de se rever o currículo dos cursos de enfermagem e a qualidade da formação do profissional, bem como investir em estratégias que favoreçam a adequação e melhora da comunicação nesses espaços.

\section{CONSIDERAÇÕES FINAIS}

Conclui-se que o número de publicações acerca da liderança do enfermeiro em emergência é pequeno e não retrata evidências fortes, consideradas como padrão ouro na prática baseada em evidências, dificultando uma discussão mais atual sobre o assunto.

\section{REFERÊNCIAS}

1. Ministério da Saúde. Portaria no 1.600, de 7 de julho de 2011. Brasília (Brasil): Ministério da Saúde; 2011.

2. Ministério da Saúde. Secretaria de Atenção à Saúde.

Regulação Médica das Urgências. Brasília (Brasil): Ministério da Saúde; 2006.

3. Secretaria de Atenção à Saúde, Ministério da Saúde. Política

Nacional de Atenção às Urgências. Brasília (Brasil): Ministério da Saúde; 2006.

4. Chen R, Sharma SK. Organizational Capabilities in Emergency Incident Response: An Empirical Examination. MWAIS

Proceedings; Paper 8; 2012. Disponível em:

http://aisel.aisnet.org/mwais2012/8.

5. Balsanelli AP, Cunha ICKO, Whitaker IY. Estilos de liderança e perfil profissional de enfermeiros em unidade de terapia

intensiva. Acta Paul Enferm. 2008;21(2):300-4.

6. Grimm JW. Effective leadership: making the difference. J of

Emerg Nurs. 2010;36(1):74-7.

7. Wong CA, Laschinger HKS, Cummings GG. Authentic
Apesar dessa escassez de artigos, é importante ressaltar que dentre os estudos levantados, duas teorias de liderança se destacaram como sendo as mais utilizadas pelos enfermeiros: a liderança situacional e a liderança transformacional. Na liderança situacional há o predomínio dos estilos E3 (compartilhar) e E4 (delegar). Ambos conferem maior autonomia ao liderado quanto às decisões a serem tomadas, sendo que o enfermeiro assume um papel de encorajador do desenvolvimento das atividades de sua equipe. Já na liderança transformacional, o espírito motivador do líder reflete em uma maior satisfação por parte da equipe de enfermagem, resultando em menor rotatividade do pessoal e melhora na qualidade da assistência ao paciente.

Para exercer uma liderança eficaz, os estudos trouxeram que é preciso praticar o autoconhecimento quanto ao estilo de liderança do enfermeiro, adequando-se às diferentes situações, bem como conhecer seus liderados e o contexto ao qual está inserido. Diversos estudos analisados abordaram a importância da intensificação do processo comunicativo nesses serviços, bem como investimento na obtenção do conhecimento e formação dos enfermeiros para o desenvolvimento da habilidade de liderança. Porém, destacam que nem sempre o conhecimento teórico garantirá uma prática de liderança eficaz.

A pesquisa apresenta como lacuna a não realização da busca em outras bases de dados que poderiam evidenciar a publicação de outros artigos primários. Os autores pretendem continuar o estudo nessa temática por meio da ampliação da busca bibliográfica, bem como da elaboração de pesquisa de campo com profissionais que atuam em serviços de urgência e emergência, uma vez que essa se constitui em ampla área de pesquisa que possibilita inúmeras explorações.

leadership and nurses' voice behaviour and perceptions of care quality. J Nurs Manag. 2010;18:889-900.

8. Taylor R. Leadership theories and the development of nurses in primary health care. Primary Health Care. 2009;19(9):40-45.

9. Giltinane CL. Leadership styles and theories. Nursing Standard. 2013;27(41):35-39.

10. Pillai $R$, Williams EA. Transformational leadership, selfefficacy, group cohesiveness, commitment, and performance. J Organizational Change Management. 2004;17(2):144-159.

11. Waugh Jr. WL, Streib G. Collaboration and Leadership for Effective Emergency Management. Public Administration Review. 2006;99(1):131-140.

12. Mendes KDS, Silveira RCCP, Galvão CM. Revisão integrativa: método de pesquisa para a incorporação de evidências na saúde e na enfermagem. Rev Texto Contexto Enferm. 2008;17(4):75864.

13. Schulman JL. What's New for 2013 MeSH®. NLM Tech Bull. 2012 Nov-Dec; (389):e5. Disponível na Internet: http://www.nlm.nih.gov/pubs/techbull/nd12/nd12 mesh.html

Rev. Eletr. Enf. [Internet]. 2014 jan/mar;16(1):211-9. Disponível em: http://dx.doi.org/10.5216/ree.v16i1.19615. - doi: 10.5216/ree.v16i1.19615. 
14. Melnyk BM, Fineout-Overholt E. Making the case for evidence-based practice and cultivating a spirit of inquiry. In: Melnyk BM, Fineout-Overholt E. Evidence-based practice in nursing \& healthcare. A guide to best practice. Philadelphia: Lippincot Williams \& Wilkins; 2011.p.3-24.

15. Wehbe G, Galvão CM. Enfermeiro de unidade de emergência: sua liderança com o pessoal auxiliar de enfermagem. Acta Paul Enferm. 2001;14(3):60-70.

16. Wehbe G, Galvão MC. Aplicação da liderança situacional em enfermagem de emergência. Rev Bras Enferm. 2005;58(1):33-8. 17. Raup GH. The impact of ED nurse manager leadership style on staff nurse turnover and patient satisfaction in academic health center hospitals. J Emer Nursin. 2008;34(5):403-9.

18. Johnson JE. Leadership in a Time of Disaster: being prepared for new age threats. J Nurs Adm. 2002;32(9):455-460.

19. Walrath JM, Tomallo-Bowman R, Maguire JM. Emergency Department: Improving Patient Satisfaction. Nurs Econ. 2004;22(2):71-74.

20. Linton J, Farrell MJ. Nurses' perceptions of leadership in an adult intensive care unit: $A$ phenomenology study. Intensive Crit Care Nurs. 2009;25(2):64-71.

21. Ritchie CY, Laschinger HKS, Wong C. The effects of emotionally intelligent leadership behaviour on emergency staff nurses 'workplace empowerment and organizational commitment. Nurs Leader. 2009;22(1):70-85.

22. Wolf L. Acuity Assignation: an Ethnographic Exploration of clinical decision making by emergency nurses at initial patient presentation. Adv Emerg Nurs J. 2010;32(3):234-246. 23. Adriaenssens J, Gucht V, Maes $S$. The impact of traumatic events on emergency room nurses: findings from a questionnaire survey. Int J Nurs Studies. 2012; 49:1411-22.

24. Johansen ML, Neweark NJ. Conflicting priorities: emergency nurses' perceived disconnect between patient satisfaction and the delivery of quality patient care. J Emerg Nursin. 2012. Disponível em:

http://www.sciencedirect.com/science/article/pii/S00991767120 $\underline{01912}$

25. Gilligan P, Bhatarcharjee C, Knight G, Smith M, Hegarty D, Shenton $A$, et al. To lead or not to lead? Prospective controlled study of emergency nurses' provision of advanced life support team leadership. Emerg Med J. 2005;22:628-32.

26. Spivak M, Smith A, Logsdon MC. Developing expert clinical nurses: Grow them, hold them and let them walk away. J Nurs Manag. 2011;19(1):92-97.

27. Bernardes A, Cecílio LCO, Évora YDM, Gabriel CS, Carvalho MB. Collective and decentralized management model in public hospitals: perspective of the nursing team. Rev Lat Am Enfermagem. 2011;19(4):1003-1010.

28. Cardoso MLAP, Ramos LH, D'Innocenzo M. Liderança Coaching: um modelo de referência para o exercício do enfermeiro-líder no contexto hospitalar. Rev Esc Enferm USP. 2011; 45(3):730-7.

29. Ministério da Saúde. Política Nacional de Educação Permanente em Saúde. Brasília (Brasil): Ministério da Saúde; 2009.

30. Strapasson MR, Medeiros CRG. Liderança transformacional na enfermagem. Rev. Bras Enferm. 2009;62(2):228-233.

31. Vizeu F. Uma aproximação entre liderança transformacional e teoria da ação comunicativa. Rev Adm Mackenzie.

2011;12(1):53-81.

32. Cummings GG. Lee $H$, MacGregor T, Davey M, Wong C,

Stafford E. Factors Contributing To Nursing Leadership: A

Systematic Review. J Health Serv Res Policy. 2008;13(4):240-8.

33. Cummings GG, Midodzi WK, Estabrooks CA. The Contribution of Hospital Nursing Leadership Styles to 30-day Patient Mortality. Nurs Research. 2010;59(5):331-339.

34. McKee MC, Driscoll C, Kelloway EK, Kelly E. Exploring

linkages among transformational leadership, workplace

spirituality and well-being in health care workers. J Manag,

Spirituality and Religion. 2011;8(3):233-255.

35. Morais AS, Melleiro MM. A qualidade da assistência de enfermagem em uma unidade de emergência: a percepção do usuário. Rev. Eletr. Enf. [Internet]. 2013 jan/mar;15(1):112-20. Disponível em: http://dx.doi.org/10.5216/ree.v15i1.15243.
36. Santos MC, Bernardes A, Gabriel CS, Évora YDM, Rocha FLR. O processo comunicativo no Serviço de Atendimento Móvel de Urgência (SAMU-192). Rev Gaúcha Enferm. 2012;33(1):69-76. 37. Wong CA, Laschinger $H$, Cummings GG, Vincent $L$, O'Connor $P$. Decisional involvment of senior nurse leaders in Canadian acute care hospitals. J Nurs Manag. 2010;18:122-133.

38. Gonzaga AR, Monteiro JK. Inteligência emocional e qualidade de vida em gestores brasileiros. Aval. Psicol. 2011;10(2):117-

127.

39. Ministério da Saúde. Diretrizes Curriculares Nacionais do Curso de Graduação em Enfermagem. Resolução CNE/CES n. 3. Brasília (Brasil): Ministério da Saúde; 2001.

40. Peres AM, Ciampone MHT. Gerência e competências gerais do enfermeiro. Texto Contexto Enferm. 2006;15(3)492-499.

41. Andrade MAC, Artmann E, Trindade ZA. Humanização da saúde em um serviço de emergência de um hospital público: comparação sobre representações sociais dos profissionais antes e após a capacitação. Cien Saúde Colet. 2011;16(1):1115-1124. 42. Dhuey E, Lipscomb S. What makes a leader? Relative age and high school leadership. Econ Educational Review. 2008;27(2):173-183.

43. Northouse PG. Leadership: theory and practice. 6 ed. Washington: Sage Publications; 2012.

44. Cristina JA, et. al. Vivências de uma equipe multiprofissional de atendimento pré-hospitalar móvel em suporte avançado de vida na assistência ao adulto em situação de parada cardiorrespiratória. Cien Enferm. 2008;14(2):97-105.

45. Santos JLG, Lima MADS. Gerenciamento do cuidado: ações dos enfermeiros em um serviço hospitalar de emergência. Rev. Gaúcha Enferm. 2011;32(4):695-702. 46. May A, Norbury J. Follow the leader. Emerg Nurs. 2007;15(4):16-21.

Artigo recebido em $10 / 08 / 12$

Aprovado para publicação em 29/06/13

Artigo publicado em 31/03/2014 